\title{
Numerical analysis of direct time-domain response of transient electromagnetic field in homogeneous half space
}

\author{
Heyua Wang , Hua Guo \\ College of science \\ Liaoning University of Technology \\ Jinzhou Liaoning China
}

\author{
Guoqian Xue \\ Institute of Geology and Geophysics \\ Chinese Academy of Sciences \\ Beijing China
}

\begin{abstract}
Near source time-domain electromagnetic field has the advantages of strong signal, great detection depth, and high accuracy, but according to the traditional transient electromagnetic field theory, the dipole approximation in near source zone will cause a bigger error. To carry out direct timedomain analytical research is one of methods to solve the problem. Under the assumption of point charge element, the paper gives direct time-domain analytic expression of transient electromagnetic field on the surface of uniform half space, and then derives direct time-domain analytic expression of transient electromagnetic field excited by square loop source. Then, on the half-space surface, made a comparison with the expression under the assumption of electric dipole source. Numerical results show that their calculation results have a little difference in the far source area but a big error in the near source area.
\end{abstract}

Keywords—component; formatting; style; styling; insert

\section{INTRODUCTION}

The classical dipole approximation has made great success in theory and practice. But for large loop device, the dipole hypothesis is not entirely valid for the detection of the whole zone. Though the dipole superposition weakened the detection error in the near source zone, it cannot be fundamentally eliminated. Nabighian gave the frequency-domain Green's function of unit point source,and through the inverse Fourier transform to obtain time-domain Green's function of full-space electrostatic field of direct current point source, and then get analytic expression of transient dipole element electromagnetic response. Because point source is placed in the origin of coordinates in the derivation, so it is unable to deal with large scale, long grounding conductor source and a large loop source device etc.

Xue Guoqiang and Yan Shu et al. proposed the research idea of direct time-domain solution of transient electromagnetic field based on current-carrying micro element of time-varying point charge, use point charge instead of dipole hypothesis, and directly derive time-domain transient electromagnetic field based on time-domain potential function. By calculating the error between static field dipole approximation solution and the exact solution, the literature gave the conclusion that there is a big error in the near zone. Zhou Nannan further calculated the error of magnetic dipole, electric dipole approximation solutions and the exact solution of current loop and current- carrying conductor without dipole hypothesis and obtained the conclusion that using the dipole approximation will bring large error in the near source region and a part of middle source region.

Xue Guoqiang and Wang Heyuan et al., by solving the Green function solution of mathematical physical equation, gave full space active wave field, and time-domain diffuse field response formula. On this basis, the literature researched problem of direct time-domain homogeneous half-space solution, derived first active wave field of upper half-space and time-domain analytic expression of reflection wave and second passive wave field of bottom half-space, but some undetermined constants have not been given,nor done comparison on the transient electromagnetic fields under the assumption of the two kind of sources. This paper deduced concrete direct time-domain analytical formula of homogeneous half space based on their work, and made a comparative analysis on transient electric field of the square loop source alongaxis andaxis direction under the assumption of two kind of sources.

\section{DiRect Time Domain ANALysis of The TrAnsient ElECTROMAgNETIC FiEld Under THE Assumption OF THE Point Charge ON THE SuRface OF THE UNIFORM HALF SPACE}

Supposeis electric fieldormagnetic field, and the two kinds of media forand ,is the conductivity, is the permeability, is the permittivity, the axis and axis are at the medium interfaces, take theupward direction as the positive direction, as shown in Fig.1.

The upper half space field meets, whereis active fist wave field, the solution is given by Green's function solution of active wave equation under the full-space non-homogeneous initial conditions,is passive secondary wave field, which is the secondary field produced by induction current in bottom half space geological body, and can obtain the analytical solution by solving homogeneous wave equation .

The electromagnetic field of the bottom half space is, the transition from passive damping wave field to diffuse field, With the help of the solution of homogeneous damping wave equation, the corresponding analytical formula can be obtained, in order to facilitate the study and calculation, we put field 
point in the axisand the axis, because the formula is complex and omitted it here.

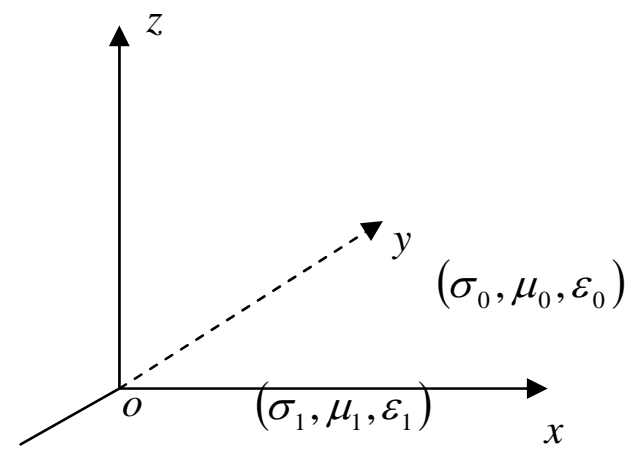

Fig. 1 The diagram of half-space medium boundary

\section{Direct Time-domain Solution of Square Loop Source UNDER TWO ASSUMPTIONS}

For square loop source device, we establish 3D coordinate system as shown in Fig. 2. When, it can be simplified into a plane coordinate system (Fig.3). The source points is located at four straight line segments, is field point. In order to facilitate research, we put the field points in the axisand axis. By the derived time-domain electromagnetic field intensity formula of homogeneous half space, we can obtain the total electric field strength at any point along the axisand the axisunder the assumption of point charge. And the analytic formula of transient time-domain electric field along the axisand axisunder the assumption of electric dipole source is a classical result], by the classical time-domain electromagnetic field intensity formula, we can obtain the total electric field strength at any point along the axisand axisunder the assumption of dipole source.

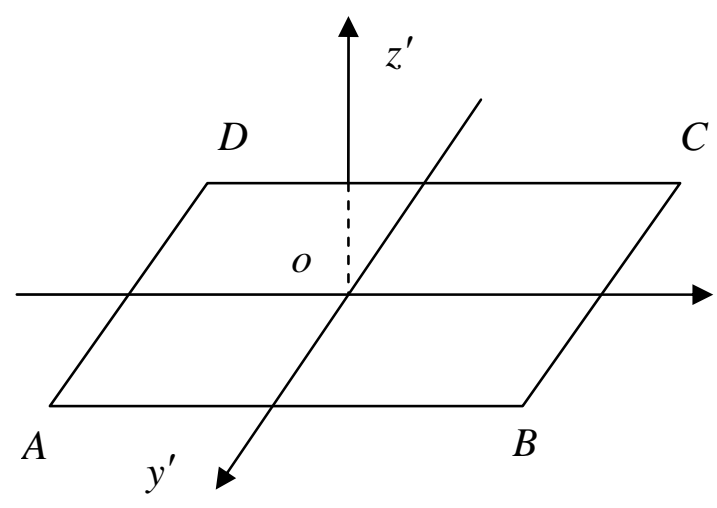

Fig. 2 TEM loop source 3D diagram

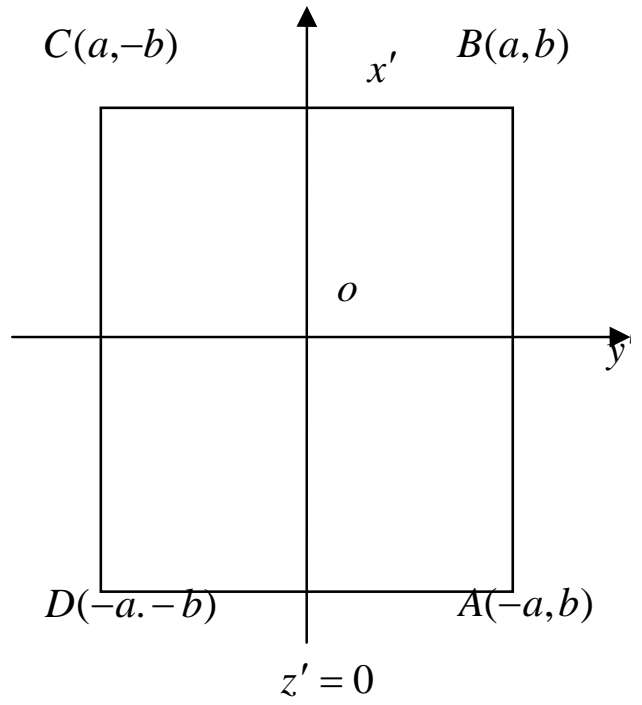

Fig. 3 TEM loop source 2D diagram

\section{NUMERICAL ANALYSIS OF TRANSIENT ELECTRIC FIELD OF SQUARE LOOP SOURCE UNDER THE ASSUMPTION OF TWO KINDS OF SOURCES}

After the derivation of transient field time-domain analytic formula of uniform half space produced by square loop source under the assumption of point charge source and electric dipole source along the direction of axis and axis, we carry out the numerical comparative analysis. Take to be approximately 500 ,m.

In practical problems, the length and the width of the square loop source may be more than $500 \mathrm{~m}$, far more than $1 \mathrm{~m}$, and the choosing field point of the far zone is at least in the tens of thousands of meters away.

Now make analysis on the calculation results of derived formula.

Analysis method: conduct numerical analysis oncomponent of the total transient electric field intensity calculated by the square loop source under the assumption of the two sources along the axis, axisin the far zone and near zone, respectively ; Similarly, conduct numerical analysis on component of the total transient electric field intensity calculated by the square loop source under the assumption of the two sources in the far zone and near zone along the axis, axis,respectively ;

The numerical results of the transient electric field values calculated by the square loop source under the assumption of point charge source and dipole source are as follows.

The numerical analysis result on component of the total transient electric field intensity calculated by the square loop source under the assumption of the two sources along the axisin the far zone as shown in Fig. 4.

The numerical analysis result oncomponent of the total transient electric field intensity calculated by the square loop source under the assumption of the two sources along the axisin the near zone as shown in Fig. 5. 


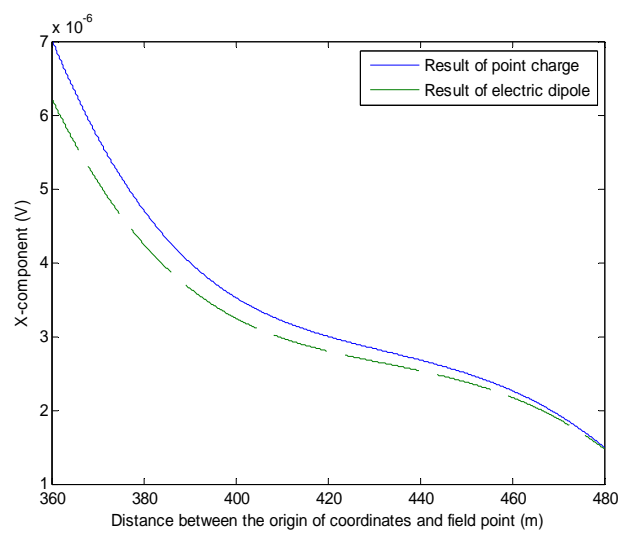

Fig. 4 Electric field value of square loop source along the axis $\mathrm{x}$ in the far region

It can be drawn according to the Fig. 4 and Fig.5 : on a homogeneous half space, the electric field strength values of the ${ }^{X}$ component value along the axial ${ }^{X}$ direction produced by square loop source under the assumption of point charge source and dipole source in the far source area is close. the transient electric field strength values of the $X_{\text {component value along }}$ the axis $y$ direction produced by square loop source in hypothesis of point charge source and dipole source in the far source area is also relatively close. In the far source area, at the point $480 \mathrm{~m}$ along the axis $X$ direction, the electric field component values produced by square loop source under the assumption of the dipole source and point source were $1.48 \times$ 10-6 and $1.50 \times 10-6$, respectively. Therefore, in the far source area, the both is equivalent.

But in the near source zone, comparing the transient electric field values of electric dipole along the axis $X$ direction under the assumption of charge source with that of point source assumption, the deviation is large, and the transient electric field values is also similar result along the axis $y$ direction, such as at $25 \mathrm{~m}$ of the near source region along the axis $y$ direction, the electric field $x$ component values produced by square loop source under the assumption of the dipole source and point source were $2.50 \times 10-4$ and $4.00 \times 10$ 4, respectively. For homogeneous half space, in the near source area, the transient electric field value of square loop source under assumption of point charge source and dipole source along the axis $x$ and the axis $y$ direction have a larger difference, the dipole approximation is no longer valid.

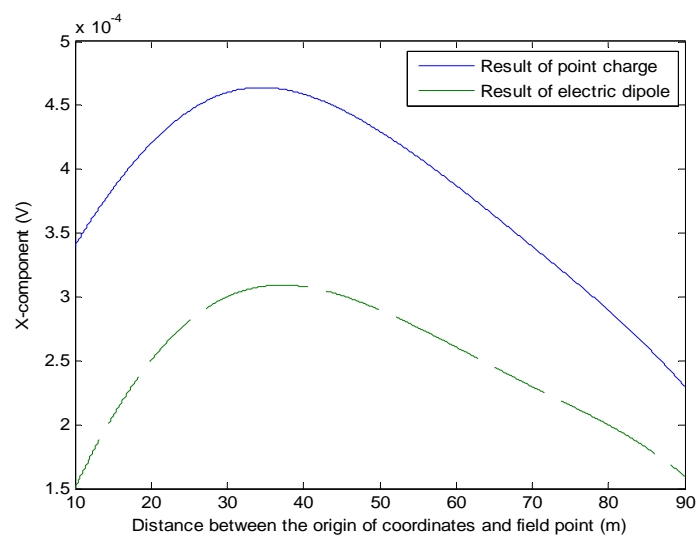

Fig. 5 Electric field value about square loop source along the axis in the near region

\section{CONCLUSIONS}

From Fig. 4 and Fig.5, in case of homogeneous half space, for the far source area, the total electric field intensity component value along the axisand the axiscalculated by square loop source under the assumption of point charge source and dipole source is comparatively close, the two quantities are equivalent, but in the near source area, comparing the calculation results of the electric field value under the assumption of electric dipole source with that under the assumption of point source, the deviation is very large. So for the near source area, square loop sources have a larger field value difference under the assumption of point charge source and dipole source, the dipole approximation is no longer valid. the direct time-domain analytical formula under point source assumption provides a theoretical basis for high precision and the detection of full source zone of transient electromagnetic field.

\section{REFERENCES}

[1] Knight J H, Raiche A P.1982. Transient electromagnetic calculations using the Gaver-Stehfest inverse laplace transform method [J]. Geophysics, 47(1): 47-50

[2] Zhou Nan-nan, Xue Guo-qiang, Wang He-yuan. 2013. Comparison of the time-domain electromagnetic field from an infinitesimal point charge and dipole source[J]. Applied Geophysics, 10(3): 349-356

[3] Poddar M. 1983. A rectangular loop source of current on multilayered earth [J]. Geophysics, 1983, 48(1): 107-109. 